\title{
Memantine prevents memory consolidation failure induced by soluble beta amyloid in rats
}

\section{Paolo Tucci ${ }^{1}$, Emanuela Mhillaj ${ }^{1}$, Maria Grazia Morgese ${ }^{1}$, Marilena Colaianna ${ }^{2}$, Margherita Zotti ${ }^{1}$, Stefania Schiavone ${ }^{3}$, Maria Cicerale ${ }^{1}$, Viviana Trezza ${ }^{4}$, Patrizia Campolongo ${ }^{5}$, Vincenzo Cuomo ${ }^{5}$ and Luigia Trabace ${ }^{1 *}$}

\author{
${ }^{1}$ Department of Experimental and Clinical Medicine, Faculty of Medicine, University of Foggia, Foggia, Italy \\ ${ }^{2}$ Department of Pathology and Immunology, University of Geneva, Geneva, Switzerland \\ ${ }^{3}$ Department of Mental Health and Psychiatry, Geneva University Hospital and University of Geneva, Geneva, Switzerland \\ ${ }^{4}$ Department of Sciences, University "Roma Tre", Rome, Italy \\ ${ }^{5}$ Department of Physiology and Pharmacology, La Sapienza, University of Rome, Rome, Italy
}

\section{Edited by:}

Katharina A. Braun

Otto-von-Guericke University,

Germany

\section{Reviewed by:}

José M. Delgado-García, University Pablo de Olavide, Spain

Bharanidharan Shanmugasundaram, Medical University of Vienna,

Austria

\section{*Correspondence:}

Luigia Trabace, Department of Experimental and Clinical Medicine,

Faculty of Medicine, University of

Foggia, Viale L. Pinto, 71121

Foggia, Italy

e-mail: luigia.trabace@unifg.it
It has been well documented that $\beta$-amyloid $(A \beta)$ peptide accumulation and aggregation in the brain plays a crucial role in the pathophysiology of Alzheimer's disease (AD). However, a new orientation of the amyloid cascade hypothesis has evidenced that soluble forms of the peptide (sA $\beta$ ) are involved in $A \beta$-induced cognitive impairment and cause rapid disruption of the synaptic mechanisms underlying memory. The primary aim of this study was to elucidate the effects of $s A \beta$, acutely injected intracerebrally (i.c.v., $4 \mu \mathrm{M}$ ), on the short term and long term memory of young adult male rats, by using the novel object recognition task. Glutamatergic receptors have been proposed as mediating the effect of $A \beta$ on synaptic plasticity and memory. Thus, we also investigated the effects of sA $\beta$ on prefrontal cortex (PFC) glutamate release and the specific contribution of $\mathrm{N}$-methyl-Daspartate (NMDA) receptor modulation to the effects of $s A \beta$ administration on the cognitive parameters evaluated. We found that a single i.c.v. injection of sA $2 \mathrm{~h}$ before testing did not alter the ability of rats to differentiate between a familiar and a novel object, in a short term memory test, while it was able to negatively affect consolidation/retrieval of long term memory. Moreover, a significant increase of glutamate levels was found in PFC of rats treated with the peptide $2 \mathrm{~h}$ earlier. Interestingly, memory deficit induced by $s A \beta$ was reversed by a NMDA-receptor antagonist, memantine $(5 \mathrm{mg} / \mathrm{kg}$ i.p), administered immediately after the familiarization trial (T1). On the contrary, memantine administered 30 min before $\mathrm{T} 1$ trial, was not able to rescue long term memory impairment. Taken together, our results suggest that an acute i.c.v. injection of sA $\beta$ peptide interferes with the consolidation/retrieval of long term memory. Moreover, such sA $\beta$-induced effect indicates the involvement of glutamatergic system, proposing that NMDA receptor inhibition might prevent or lead to the recovery of early cognitive impairment.

Keywords: soluble beta amyloid, glutamate, short term memory, long term memory, memantine

\section{INTRODUCTION}

Extensive research on the neurotoxic role of beta amyloid $(\mathrm{A} \beta)$ in its fibrillar form has demonstrated that it accumulates in the brain as extracellular insoluble plaques around neurons and glia (Kowalewski and Holtzman, 1999; Mucke and Selkoe, 2012). Later, this view changed when it was recognized that in Alzheimer's disease (AD) patients, cognitive impairment is poorly correlated with counts of "senile plaques" in cerebral gray matter either in patients (Blennow et al., 1996; Berg et al., 1998; Giannakopoulos et al., 2003, 2009) or in animal models (Puoliväli et al., 2002; Christensen et al., 2008; Watanabe et al., 2009; Zhang et al., 2012). More recently, in support of this, the initial etiopathogenetic hypothesis has been questioned by the failure of several clinical trials testing drugs targeting $A \beta$ accumulation in the brain (Mangialasche et al., 2010).

Instead, a new orientation of the amyloid cascade hypothesis appeared and more recent studies suggested that a stronger interrelationship exists between the presence of soluble beta amyloid $(\mathrm{s} A \beta)$ and dementia severity. Soluble $\mathrm{A} \beta$ oligomers were recently reported to be dramatically increased in the soluble fraction of AD brain extracts (Sokolow et al., 2012), and they have been found to correlate with disease progression or decline in synaptic density in AD patients (Lue et al., 1999; McLean et al., 1999). In this scenario, soluble oligomeric, globular and protofibrillar amyloid species, rather than fibrils, could be considered the first toxic species and are hypothesized to contribute to synaptic failure and early cognitive loss in $\mathrm{AD}$ (Arendt, 2009). In this regard, the 
role of $\mathrm{sA} \beta$ in the pathophysiology of $\mathrm{AD}$ still remains a matter of intense research at present. Interestingly, impaired neuronal functions have been directly attributed to soluble oligomeric forms of $A \beta$, even before the appearance of overt signs of neurotoxicity (Lesné et al., 2006; Haass and Selkoe, 2007). Moreover, $\mathrm{sA} \beta$ has been found to cause rapid disruption of the synaptic mechanisms underlying memory. It has been demonstrated that sA $\beta$ oligomers, directly extracted from the cerebral cortex of subjects with $\mathrm{AD}$, were able to disrupt the memory of a learned behavior in normal rats (Shankar et al., 2008). However, very few studies have focused on the investigation of the effects of defined sequences of sA $\beta$ on memory. In this regard, transgenic mice models of $\mathrm{AD}$, although covering several aspects of the disease, do not permit an accurate identification of $A \beta$ species responsible of memory loss, being the peptide expressed either in soluble or insoluble forms in the brain. This characteristic does not allow to determine which specific $A \beta$ species is responsible for the detrimental effects. On the other hand, peptide injection in the rat's brain might be considered as a valid alternative to transgenic animals to evaluate the effects of an increase of $\mathrm{sA} \beta$ species in the brain without presence of plaques. Then, to date, the comprehension of the specific role of sA $\beta$ on different temporal forms of memory remains an urgent need. To further investigate the specific effects of sA $\beta$, we recently developed a simple and reliable rat model of memory impairment induced by an acute i.c.v. injection of sA $\beta 1-42$ (Trabace et al., 2007; Colaianna et al., 2010). We demonstrated that $\mathrm{sA} \beta$ significantly affected behavior that rely on stressful conditions. In particular, $s A \beta$-treated rats were not able to retrieve the learned behavior, fear conditioned, in a passive avoidance task (Morgese et al., 2014).

In order to evaluate if sA $\beta$-treated rats may differently respond to behavioral cognitive tasks that do not involve stressful situations, we used an animal paradigm that is not demanding for the animals, but is based on the natural propensity of rats to explore novel situations. A simple and reliable task widely used for the investigation of memory is the one-trial novel object recognition (NOR) test. This procedure typically consists of three phases: habituation, familiarization, and finally the test phase. Rats naturally tend to approach and to explore novel over familiar objects. Such task relies on spontaneous animal behavior without the need neither for positive reinforcers nor for stressor elements, such as water or food deprivation, electric foot-shock or aversive environments. In particular, the ability of recognizing a previously presented stimulus varies according to the delay between the familiarization and the test phase. Based on this, the NOR test is very useful to study short-term and long-term memory (Ennaceur and Delacour, 1988; Taglialatela et al., 2009). This is possible by manipulating the amount of time rats must retain memory of the sample objects presented during the familiarization phase. In particular, short term memory is represented by the process that maintains a representation of information for a short period of time, and it is available for posterior use, while the long-term memory requires stabilization to persist. This process implies a reorganization of the already established memories, allowing then incorporation of new information (Clarke et al., 2010). In this regard, early studies carried out in senescence-acceleratedprone 8 (SAMP8) mouse strain, presenting cognitive deficiencies that could be related to the accumulation of amyloid aggregates, have shown a functional alteration in the hippocampalprefrontal circuits which is related to the object recognition deficit (Lopez-Ramos et al., 2012).

The current study was designed to address on which phase of the memory process $\mathrm{sA} \beta$ exerts its effects. We evaluated the effects of sA $\beta$, acutely injected intracerebrally, on the short term and long term memory of young adult male rats. Since the training-testing interval is extremely informative regarding the mechanism of action underlying the type of memory impairment, we chose two different experimental paradigms, especially well-suited to study short term memory ( 1 min retention interval, NOR1) and long term memory ( $24 \mathrm{~h}$ retention interval, NOR2).

Literature data point out that soluble forms of the peptide, in particular oligomers ranging from dimers and trimers to dodecamers, are involved in $\mathrm{A} \beta$-induced cognitive impairment and are capable of blocking long-term potentiation (LTP) and the reversal of long term depression (LTD; Lambert et al., 1998; Wilcox et al., 2011). Moreover, in $\mathrm{A} \beta$-based model of $\mathrm{AD}$, it has been found a predominant susceptibility of glutamatergic synapses (Canas et al., 2014). In addition, a direct application of sA $\beta$ oligomers or fragments to cultured hippocampal neurons was found to rapidly trigger sustained calcium entry through $\mathrm{N}$-methyl-D-aspartate (NMDA) receptors that was prevented by antagonists of NMDA receptors (Kelly and Ferreira, 2006; De Felice et al., 2007) and to enhance NMDA-evoked cell firing rate (Molnár et al., 2004). Moreover, fragments of $\mathrm{A} \beta$ have been shown to bind glycine and glutamate recognition sites on NMDA receptors (Cowburn et al., 1997). Therefore, a further aim of the present study was to examine the effects of $s A \beta$ on glutamate release and the specific contribution of NMDA receptor modulation to the cognitive deficits associated with $s A \beta$ administration. To this end, we tested the effects of NMDA receptor inhibition on memory disruption in rats i.c.v. injected with $\mathrm{sA} \beta$.

\section{MATERIALS AND METHODS ANIMALS}

All experiments were conducted in young male Wistar rats (Harlan S. Pietro al Natisone, Udine, Italy) weighing 250-300 g. They were group-housed at constant room temperature $\left(22 \pm 1^{\circ} \mathrm{C}\right)$ and relative humidity $(55 \pm 5 \%)$ under a 12 -h light/dark cycle (lights on from 7:00 AM to 7:00 PM) for at least 7 days before the experiments. Food and water were available ad libitum. Procedures involving animals and their care were conducted in conformity with the institutional guidelines in compliance with national (D.L. 116/92) and international laws and policies (EEC Council Directive 2010/63/EU; Guide for the Care and Use of Laboratory Animals, U.S. National Research Council, 1996). All efforts were made to minimize the number of animals used and their suffering.

\section{SURGERY}

Surgery procedures were performed as previously described (Colaianna et al., 2010). Briefly, rats were anesthetized with $3.6 \mathrm{ml} / \mathrm{kg}$ Equithesin intraperitoneally (i.p.; composition: $1.2 \mathrm{~g}$ sodium pentobarbital; $5.3 \mathrm{~g}$ chloral hydrate; $2.7 \mathrm{~g} \mathrm{MgSO}_{4} ; 49.5 \mathrm{ml}$ 
propylene glycol; $12.5 \mathrm{ml}$ ethanol and $58 \mathrm{ml}$ distilled water) and secured in a stereotaxic frame (David Kopf Instruments, Tujunga, CA, USA). The skin was shaved, disinfected and cut with a sterile scalpel to expose the skull in order to perform the procedures described below.

\section{SOLUBLE AB1-42 ADMINISTRATION}

The sA $\beta$ peptide was obtained from Tocris (Bristol, UK). All solutions were freshly prepared. Peptide was dissolved in sterile double-distilled water (vehicle) at a concentration of $4 \mu \mathrm{M}$ as previously described (Colaianna et al., 2010). On the day of surgery, bilateral 23 gauge guide cannulae were implanted using the following coordinates relative to Bregma: $\mathrm{AP}=-0.5 \mathrm{~mm}, \mathrm{ML}$ $=+1.2 \mathrm{~mm}, \mathrm{DV}=-3.0 \mathrm{~mm}$ with the incisor bar set at $-3.3 \mathrm{~mm}$, according to a stereotaxic atlas (Paxinos and Watson, 1998). The day after surgery procedure, intraventricular injections were made using injection needles (30-gauge stainless steel tubing; Cooper's Needles, Birmingham, UK) which extended an additional $0.2 \mathrm{~mm}$ below the guide cannulae (total depth $3.2 \mathrm{~mm})$. Soluble A $\beta(5 \mu \mathrm{l})$ was delivered through a $25 \mu \mathrm{l}$ Hamilton microsyringe at $2 \mu \mathrm{l} / \mathrm{min}$ infusion rate for duration of $2.5 \mathrm{~min}$. Control rats were injected with vehicle only, because reverse sA $\beta 42-1$, used in preliminary experiments, had no effect on the measured parameters and was indistinguishable from vehicle alone (unpublished observations). The injection needle was left in place for additional $5 \mathrm{~min}$ to prevent reflux of the solution. The injection placement of needle track was verified at the time of dissection. All experimental procedures were performed $2 \mathrm{~h}$ after i.c.v. administration (shamoperated or $s A \beta$-treated groups).

\section{OTHER CHEMICALS}

Memantine hydrochloride (3,5-dimethyl-1-adamantanamine hydrochloride, 3,5-dimethylamantadine hydrochloride) was purchased from Sigma-Aldrich Chemical Co. (Milan, Italy). It was dissolved in $1 \mathrm{ml} / \mathrm{kg}$ of $0.9 \% \mathrm{NaCl}$ (saline) and administered intraperitoneally at a dose of $5 \mathrm{mg} / \mathrm{kg}$.

\section{NOVEL OBJECT RECOGNITION (NOR) TEST}

During the NOR test rats were submitted to two habituation sessions where they were allowed $5 \mathrm{~min}$ to explore the apparatus (circular arena, $75 \mathrm{~cm}$ diameter).

The NOR1 test was performed according to Giustino et al. (1996). Briefly, $24 \mathrm{~h}$ after last habituation, a session of two 3-min trials separated by a 1-min intertrial interval (retention interval) was carried out (Figure 1A).

The NOR2 test was performed according to Balducci et al. (2010). Briefly, $24 \mathrm{~h}$ after last habituation, a session of two 10-min trials separated by a $24 \mathrm{~h}$ intertrial interval (retention interval) was carried out (Figure 1B). In both experimental conditions (NOR1 and NOR2), 2 h before the first trial (T1, training session), rats received i.c.v injection of $\mathrm{sA} \beta$ solution or vehicle. Then, animals were exposed to two identical objects (white glasses or light bulbs). During the second trial (T2, testing session), rats were exposed to one familiar (F) object and the second was replaced by a new object $(\mathrm{N})$ differently shaped. In a different session using the NOR2 protocol, memantine was administered i.p. ( $5 \mathrm{mg} / \mathrm{kg})$ $30 \mathrm{~min}$ before or immediately after T1 trial.
In both protocols, during each trial, objects were placed in an equidistant position between the center and the wall of the arena. From rat to rat, the position of the two objects was counterbalanced and randomly permuted during T2. At the beginning of each trial, rats were placed near the center of the arena with their heads oriented in the opposite direction to the objects. Exploration of the objects was defined as sniffing or touching the object with the nose. Turning around or sitting on the object was not considered as exploration (Ennaceur and Delacour, 1988). Object exploration was quantified as: exploratory activity, total time spent exploring both objects during each trial (T1 and T2); index of discrimination expressed as ratio between the net time spent exploring the new $(\mathrm{N}-\mathrm{F})$ over the total exploration time $(\mathrm{N}-\mathrm{F} / \mathrm{N}+\mathrm{F})$. Objects and arena were carefully cleaned between each session to avoid olfactory confounding stimuli. Preliminary tests showed that none of the objects used in our experiments evoked innate preference.

\section{MICRODIALYSIS PROCEDURE}

Animals were deeply anesthetized as reported above and placed on a stereotaxic apparatus (David Kopf Instruments, Tujunga, CA). One horizontal custom-constructed microdialysis probe (AN69 HospalS.p.A; $20 \mathrm{kDa}$ cut-off, $6 \mathrm{~mm}$ length) was implanted aiming at the prefrontal cortex (PFC). The coordinates, measured from the interaural line, were $\mathrm{AP}=+10.7 \mathrm{~mm}$ and $\mathrm{DV}=+8.0 \mathrm{~mm}$ according to the atlas (Paxinos and Watson, 1998). The microdialysis probe was fixed to the skull with stainless steel screws and methylacrylic cement. Twenty four hours after surgery, the microdialysis probe was perfused with artificial CSF solution $\left(\mathrm{NaCl} 145 \mathrm{mmol} / \mathrm{L}, \mathrm{KCl} 2.7 \mathrm{mmol} / \mathrm{L}, \mathrm{CaCl}_{2} 2 \mathrm{H}_{2} \mathrm{O} 1.2\right.$ $\mathrm{mmol} / \mathrm{L}, \mathrm{MgCl}_{2} 6 \mathrm{H}_{2} \mathrm{O} 1 \mathrm{mmol} / \mathrm{L}, \mathrm{Na}_{2} \mathrm{HPO}_{4} 2 \mathrm{mmol} / \mathrm{L}, \mathrm{pH}$ 7.4) at a constant flow rate of $2 \mu \mathrm{l} / \mathrm{min}$. Perfusates were collected every $20 \mathrm{~min}$ into mini-vials. After a wash-out period of $2 \mathrm{~h}$, four samples were collected to determine the baseline levels of glutamate (no more than 10\% difference among four consecutive samples). Glutamate concentrations were detected and quantified by high performance liquid chromatography (HPLC).

\section{QUANTIFICATION OF GLUTAMATE BY HPLC}

Glutamate concentrations were determined by HPLC using ODS3 column $(150 \times 4.6 \mathrm{~mm}, 3 \mu \mathrm{m}$; INERTSIL $)$ with fluor escence detection after derivatization with ophthalaldehyde/mercaptopropionic acid (emission length, $4.60 \mathrm{~nm}$; excitation length, $3.40 \mathrm{~nm}$ ). The mobile phase gradient consisted of $50 \mathrm{mM}$ sodium acetate buffer, $\mathrm{pH}$ 6.95, with methanol increasing linearly from 2 to $30 \%(\mathrm{v} / \mathrm{v})$ over $40 \mathrm{~min}$. The flow rate was maintained by a pump (JASCO, Tokyo, Japan) at $0.5 \mathrm{ml} / \mathrm{min}$. Results were analyzed by Borwin software (version 1.50; Jasco) and substrate concentration was expressed as $\mu \mathrm{M}$.

\section{STATISTICAL ANALYSIS}

All statistical analyses were performed using Graph $\operatorname{Pad}^{\circledR} 5.0$ for Windows. Data were tested for normality by the selection of parametric and non-parametric tests. Data were analyzed by a Two-way analysis of variance for repeated measures (Two-way RM ANOVA) followed by a Bonferroni's multiple comparison 
A

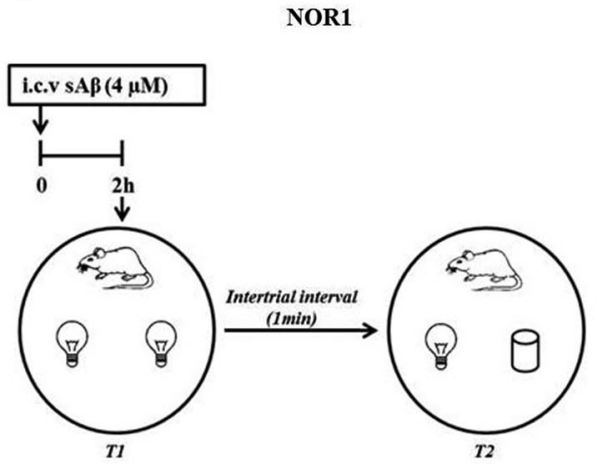

B

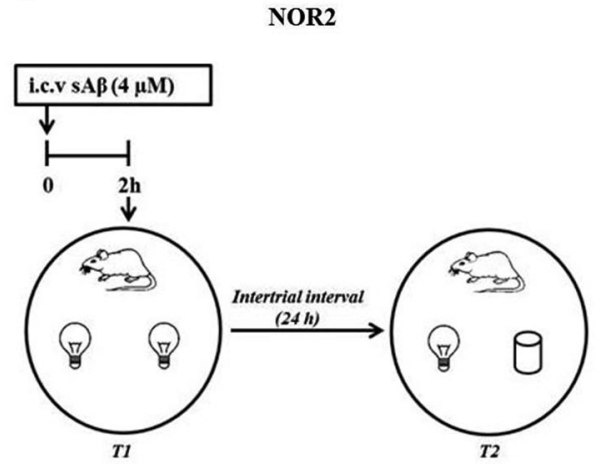

FIGURE 1 | Schematic representation of experimental procedure (A) NOR1 protocol according to Giustino et al. (1996); (B) NOR2 protocol according to Balducci et al. (2010)

test or Student's $t$-test, as required. Differences were considered significant only when $P$-values were less than 0.05 .

\section{RESULTS}

\section{EFFECTS OF ACUTE I.C.V. INJECTION OF SA $\beta$ ON SHORT TERM MEMORY (NOR1)}

Our data showed that either sham- or sA $\beta$-treated rats exhibited normal performance in the NOR1 test, with a significant difference in total time spent exploring the new object rather than the familiar one during the test (Two-way RM ANOVA followed by Bonferroni's multiple comparisons test: $F t_{(1,15)}=41.54$, $P<0.0001$; Figure 2A). Moreover, the preference between the different objects (discrimination index) during testing did not change significantly between experimental groups showing that $\mathrm{sA} \beta$, injected $2 \mathrm{~h}$ before training, did not affect short term memory for the familiar object ( $t$-test: n.s.; Figure 2B). As shown in Figure 2C, no statistical differences were found in total exploration time, during both trials (T1 and T2), between sham and sA $\beta$-treated rats ( $t$-test: n.s.).

\section{EFFECTS OF ACUTE I.C.V. INJECTION OF sA $\beta$ ON LONG TERM MEMORY (NOR2)}

As shown in Figure $\mathbf{3 A}$, sA $\beta$-treated rats failed to acquire the object recognition memory. Statistical analysis revealed a significant difference in exploratory activity between the experimental groups (Two-way RM ANOVA: $F t_{(1,16)}=28.52, P<0.0001$ ). In particular, $s A \beta$-treated rats were not able to recognize the novel object, while sham-operated animals preferentially explored the novel rather than the familiar one (Bonferroni's post hoc test: $P<0.001)$. Accordingly, the discrimination index was significantly lower than controls ( $t$-test: $P<0.05$; Figure 3B). No differences in total exploration time were found between experimental groups ( $t$-test: n.s.; Figure 3C).

\section{EFFECTS OF MEMANTINE ON LONG TERM MEMORY IMPAIRMENT INDUCED BY ACUTE I.C.V. INJECTION OF SA $\beta$}

To establish whether the memory deficit observed in the NOR2 protocol was dependent on glutamatergic modulation, rats were treated with memantine $(5 \mathrm{mg} / \mathrm{kg}$ i.p.) or vehicle immediately after the 10 min training session (T1). Twenty-four hours later, rats were subjected to the test phase, as described above. As shown in Figure 4A, a clinically relevant dose of memantine was able to prevent the long term memory impairment induced by sA $\beta$ (Two-way RM ANOVA followed by Bonferroni's multiple comparisons test: $\left.F t_{(1,14)}=23.34, P<0.001\right)$. Then, while rats injected with $\mathrm{sA} \beta$ did not discriminate between the familiar and the new object, the inhibition of NMDA receptors prevented this memory deficit ( $t$-test: n.s.; Figure 4B). No differences in total exploration time were found between experimental groups (Figure 4C).

Interestingly, when memantine was administered $30 \mathrm{~min}$ before the familiarization phase (T1), sA $\beta$-injected rats, tested $24 \mathrm{~h}$ later, remained cognitively impaired (Two-way RM ANOVA followed by Bonferroni's multiple comparisons test: $F t_{(1,10)}=25.13, P<0.001$; Figure 5A). Accordingly, the discrimination index was significantly lower than control $(t$-test: $P<0.05$; Figure 5B). Total exploration time was not modified between experimental groups ( $t$-test: n.s.; Figure 5C). The treatment with memantine alone did not affect memory.

\section{EFFECTS OF ACUTE I.C.V. INJECTION OF sA $\beta$ ON GLUTAMATE LEVELS}

Glutamate basal levels were measured in microdialysis fluid $2 \mathrm{~h}$ after i.c.v. administration of $\mathrm{sA} \beta$ or vehicle in PFC, no more than $10 \%$ difference among sample was found, then the data obtained from four consecutive samples per animal were averaged, and mean value from $n$ animals per group was determined.

As shown in Figure 6, extracellular levels of glutamate were significantly increased in PFC of sA $\beta$-treated animals compared to control $(t$-test: $P<0.05)$.

\section{DISCUSSION}

Our results show for the first time that an acute i.c.v. injection of sA $\beta$ peptide, during the process of long term memory formation, did not disrupt memory storage but, when the information was properly stored, it acutely interfered with its consolidation/retrieval, and such effect was prevented by NMDA receptor inhibition. The possibility to mimic the detrimental action of sA $\beta$ peptides in vivo provides a very interesting tool for understanding 
A familiar object

novel object

B
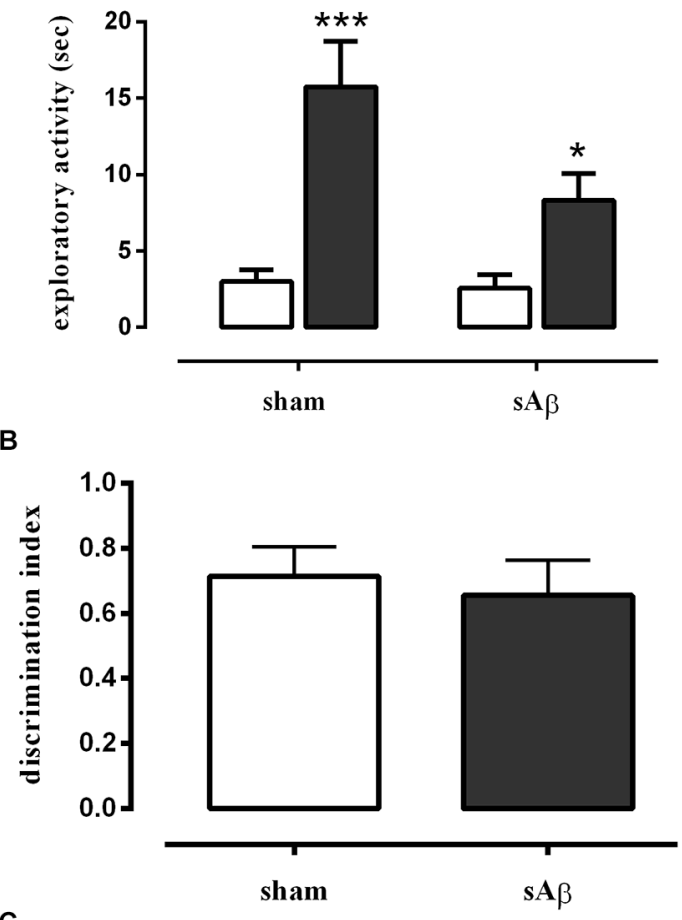

C

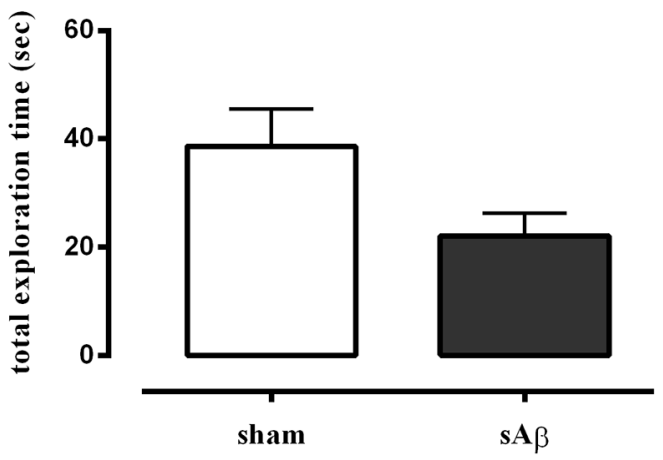

FIGURE 2 | (A) Exploratory activity of novel and familiar objects during T2, (B) discrimination index and (C) total exploration time in NOR1 test. Animals received i.c.v. injection of vehicle $(5 \mu \mathrm{l}$; sham) or sA $(4 \mu \mathrm{M}, 5 \mu \mathrm{l})$ $2 \mathrm{~h}$ before training (T1) and tested $1 \mathrm{~min}$ after (T2). Data are expressed as mean \pm SEM of total time in $\mathrm{s}(n=8$ sham; $n=9 \mathrm{sA} \beta$-treated group). (Two-way RM ANOVA followed by Bonferroni's multiple comparisons test ${ }^{* *} P<0.0001$ vs. familiar object in sham group and ${ }^{*} P<0.05$ vs. familiar object in $\mathrm{sA} \beta$-treated group).

their mechanism of action. However, since a single species of $\mathrm{A} \beta$ is responsible for the complex dysregulation of intracellular signaling pathways that takes place in $\mathrm{A} \beta$-mediated effects, it is reasonable to expect that distinct $A \beta$ assemblies may differentially affect specific pathways underlying synaptic dysfunction (Mucke and Selkoe, 2012). Moreover, given that A $\beta$ oligomeric species act on independent targets, it seems of particular interest to understand the biological effects of a particular assembly form.
A familiar object novel object

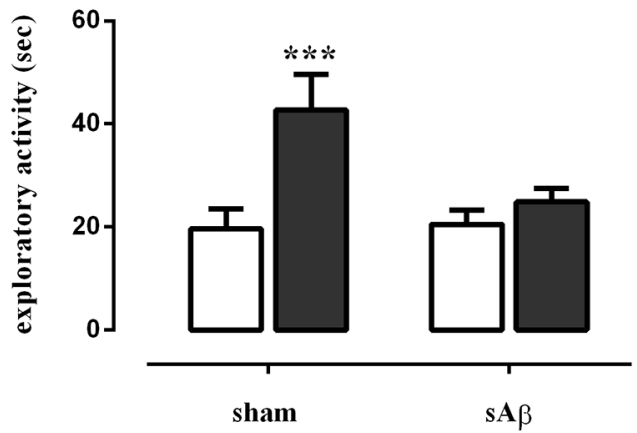

B

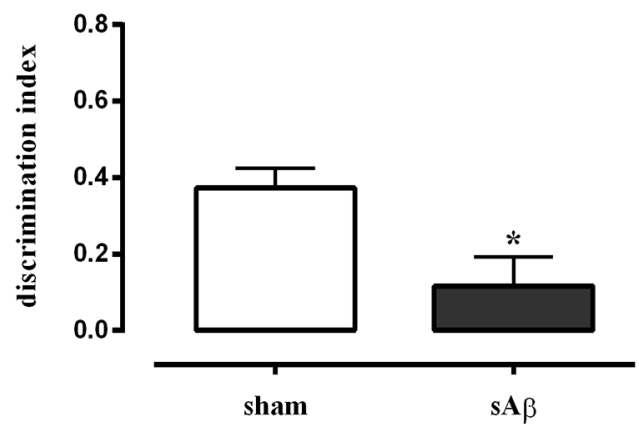

C

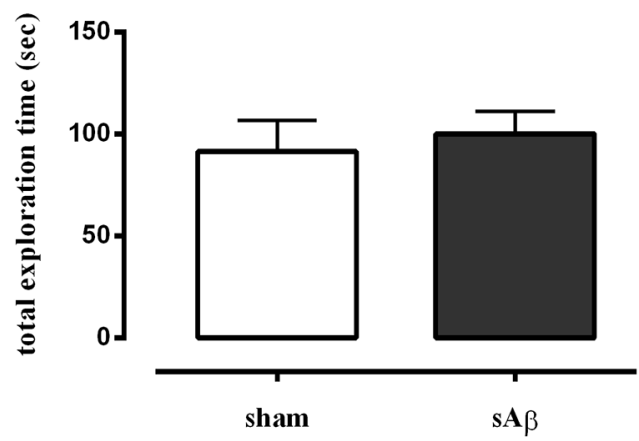

FIGURE 3 | (A) Exploratory activity of novel and familiar objects during T2, (B) discrimination index and (C) total exploration time in NOR2 test. Animals received i.c.v. injection of vehicle $(5 \mu \mathrm{l}$; sham) or $\mathrm{sA} \beta(4 \mu \mathrm{M}, 5 \mu \mathrm{l})$ $2 \mathrm{~h}$ before training ( $\mathrm{T} 1$ ) and tested $24 \mathrm{~h}$ later (T2). Data are expressed as mean \pm SEM of total time in $\mathrm{s}$ ( $n=6$ sham; $n=12 \mathrm{sA} \beta$-treated group). (Two-way RM ANOVA followed by Bonferroni's multiple comparisons test *** $P<0.001$ vs. familiar object; $t$-test ${ }^{*} P<0.05$ vs. sham).

Unlike $A \beta$ assemblies purified from brain's patients, commercial available $A \beta$ fragments are considered useful tools as they are chemically defined, and can be easily characterized, from a structural point of view, by using several techniques, ranging from western blot (Jin et al., 2011) to nuclear magnetic resonance (Yu et al., 2009) and electrophoresis (Wiltfang et al., 1997). However, one of the best and most informative technique is atomic force microscopy (AFM; Kowalewski and Holtzman, 1999; Balducci et al., 2010), which provides a high-resolution image at the molecular level revealing details of protein structures. By using 
A familiar object novel object

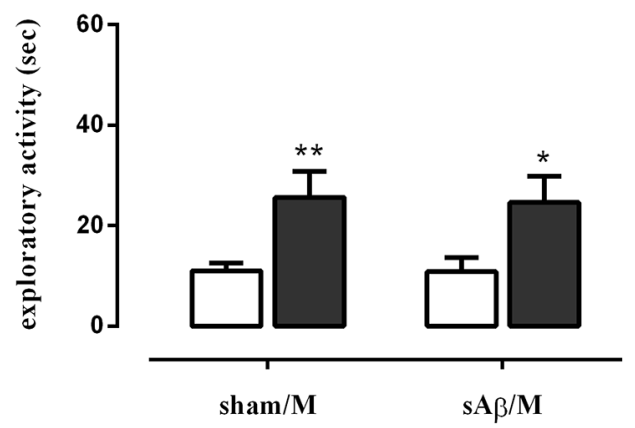

B

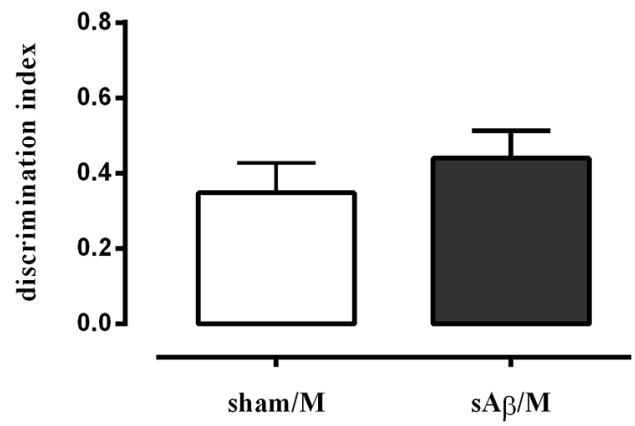

C

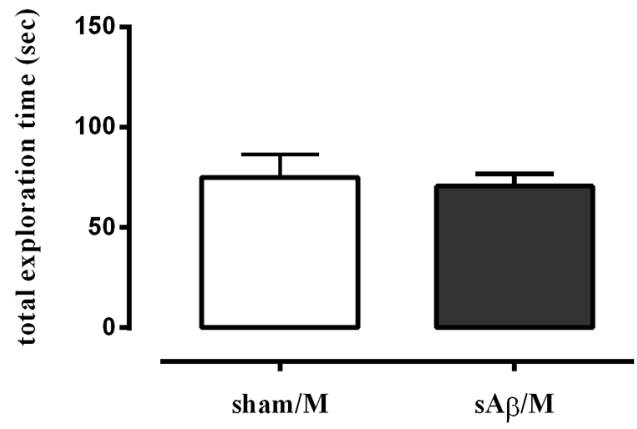

FIGURE 4 | (A) Exploratory activity of novel and familiar objects during T2, (B) discrimination index and (C) total exploration time in NOR2 test. Animals received i.c.v. injection of vehicle $(5 \mu \mathrm{l}$; sham) or sA $(4 \mu \mathrm{M}, 5 \mu \mathrm{l})$ $2 \mathrm{~h}$ before training (T1) and tested $24 \mathrm{~h}$ later (T2). Memantine ( $5 \mathrm{mg} / \mathrm{kg}$ ) was given immediately after a $10 \mathrm{~min}$ training. Data are expressed as mean \pm SEM of total time in $\mathrm{s}(n=8 \mathrm{sham} / \mathrm{M} ; n=8 \mathrm{sA} / \mathrm{M})$ ). (Two-way RM ANOVA followed by Bonferroni's multiple comparisons test ${ }^{*} P<0.01$ and ${ }^{*} P<$ 0.05 vs. familiar object).

$\mathrm{AFM}$, we precisely characterized $\mathrm{sA} \beta$ fragments and, to further confirm the conformation of the peptide, we used the transmission electronic microscopy (TEM); both techniques enabled us to feature the types of sA $\beta$ fragments, with no fibrils being present in the injected solution (our unpublished data, Campagna et al., 2011). Moreover, in our study, the use of defined synthetic sA $\beta$ preparations removed unknown factors, which can be present in cells and brain extracts or cerebrospinal fluid that could mask or exacerbate their effects. From a behavioral point of view and
A familiar object novel object

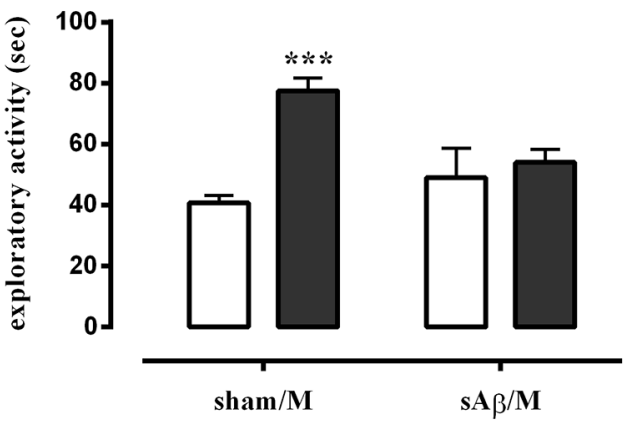

B

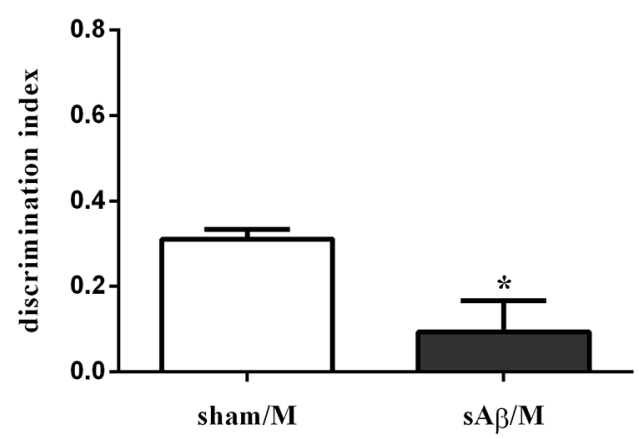

C

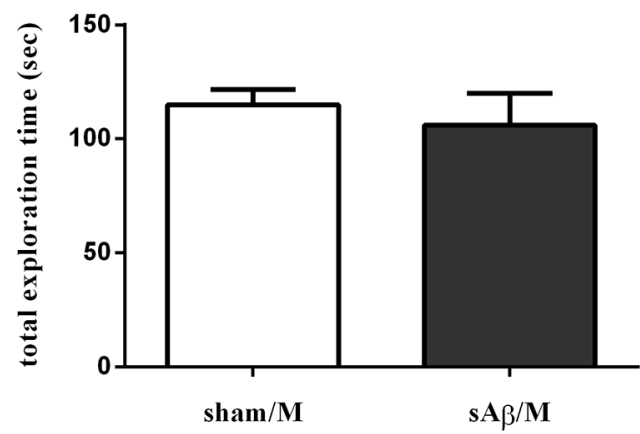

FIGURE 5 | (A) Exploratory activity of novel and familiar objects during T2, (B) discrimination index and (C) total exploration time in NOR2 test. Animals received i.c.v. injection of vehicle $(5 \mu \mathrm{l}$; sham) or sA $(4 \mu \mathrm{M}, 5 \mu \mathrm{l})$ $2 \mathrm{~h}$ before training (T1) and tested $24 \mathrm{~h}$ later (T2). Memantine ( $5 \mathrm{mg} / \mathrm{kg}$ ) was given 30 min before training. Data are expressed as mean \pm SEM of total time in $\mathrm{s}(n=6 \mathrm{sham} / \mathrm{M} ; n=6 \mathrm{sA} / \mathrm{M})$. (Two-way RM ANOVA followed by Bonferroni's multiple comparisons test ${ }^{* * *} P<0.01 \mathrm{vs}$. familiar object, t-test ${ }^{*} P<0.05$ vs. sham). 


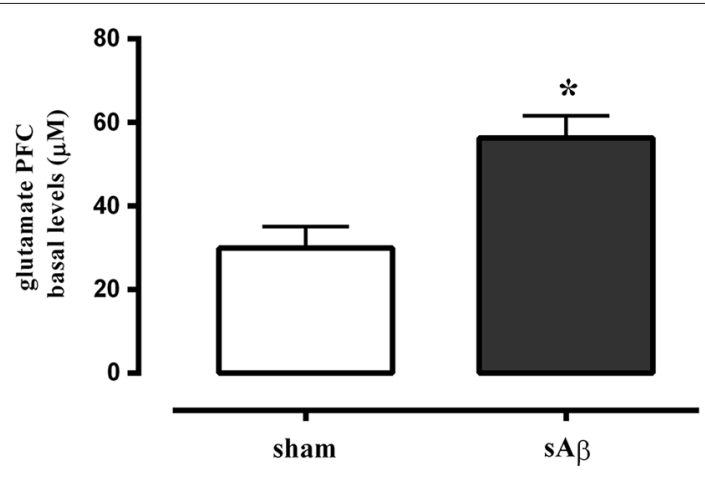

FIGURE 6 | Quantitative analysis of basal extracellular glutamate levels in PFC of rats $2 \mathrm{~h}$ after i.c.v. injection of vehicle ( $5 \mu \mathrm{L}$; sham) or sA $\boldsymbol{\beta}$

(4 $\mu \mathbf{M}, \mathbf{5} \mu \mathbf{l})$. Data are expressed as mean $\pm \operatorname{SEM}(n=6$ sham, $n=4 \mathrm{sA} \beta)$. (Student $t$-test $\left.{ }^{*} P<0.05\right)$.

In particular, results of NOR paradigm are influenced by the manipulation of the amount of time required for the intertrial interval (Taglialatela et al., 2009). In the present study, two variants of NOR test were used to investigate the effects of sA $\beta$ on short and long term memory. During the NOR1, animals were exposed to the test phase $1 \mathrm{~min}$ after the familiarization phase. During testing, a novel object needs to be detected and encoded, while a familiar object needs to be updated. In these experimental conditions, rats treated with sA $\beta 2$ h before behavioral investigation, exhibited normal performance during the test and remained cognitively intact. In these animals, when novel and familiar stimuli were present together, the novel stimulus was more explored until novelty was lost. Indeed, the preference for the novel object means that presentation of the familiar one was already present in their memory, indicating that during the NOR1, sA $\beta$ did not affect this short term memory. Then, in our animal model, sA $\beta$ injected i.c.v. before the acquisition of the information (familiarization phase) did not affect the information being encoded. These results confirmed our previous investigations where we demonstrated that, in the context of normal levels of general motor activity, sA $\beta$ did not alter the ability of rats to differentiate between a familiar and a novel object, in a short term memory test, even when the test was performed 7 days after i.c.v. injection (Colaianna et al., 2010). However, memory relies on a set of processes by which information is encoded, consolidated, and retrieved. In addition, when a certain memory is recovered in the presence of novelty, it is set into an unstable state, during which its retention can be either enhanced or impaired.

Over the next hours, memories require stabilization to persist and are consolidated over time into long term memories, and once in that state, they remain fixed. This processing memory is involved in the reorganization of the already formed memories, allowing then incorporation of new information (Clarke et al., 2010). Based on this, although the NOR1 behavioral protocol could not permit to assess sA $\beta$ effects on consolidation or recall processes, it seems that the peptide does not affect short term memory. Thus, we modified the retention interval, which is the amount of time the rat must retain the memory of the two identical objects presented during the training session prior to the testing session. Thus, the rat's ability to acquire the object recognition memory by using an intertrial interval of $24 \mathrm{~h}$ was tested. Interestingly, in these experimental conditions (NOR2), when sA $\beta$-treated rats were re-exposed to either the familiar or the novel object, they did show significant difference between groups in time spent for exploration of both objects, meaning that animals were unable to remember the object previously investigated. These data suggest that, when the information was properly encoded, as demonstrated by using the NOR1 task, sA $\beta$ did not disrupt memory storage but acutely interfered with its consolidation/retrieval. Actually, disruption of memory formation results from interference with key biological mechanisms underlying the stabilization of memory.

Depending on the time when the disturbance occurs in this biological activity, a specific phase of memory formation might be influenced. The delay-dependent decrease in memory recognition we observed might result from a decay in memory of the familiar object. Thus, one hypothesis that could account, at least in part, for these results is that $\mathrm{sA} \beta$ was able to induce anterograde amnesia, namely the inability to learn new information, although this hypothesis requires further investigation. It is known that patients with this form of amnesia preserve intact the capacity to retain small amounts of information over short periods of time but are dramatically impaired in their ability to form long-term memories (Markowitsch and Staniloiu, 2012). Thus, in our animal model, a single i.c.v. injection of sA $\beta$ impaired memory consolidation/retrieval within $24 \mathrm{~h}$, suggesting that the peptide interferes with the synaptic activity, which is essential for the stabilization of new memories (Mayford et al., 2012). Indeed, if the memory was properly consolidated in the rats, then it should have been retrievable during the test phase, as seen in controls. Thus far, there are only few in vivo studies of the involvement of sA $\beta$ in memory alterations in rats (Cleary et al., 2005; Lesné et al., 2006; Poling et al., 2008; Shankar et al., 2008). As demonstrated by LopezRamos et al. (2012), SAMP8 mice showing cognitive dysfunctions probably related to amyloid aggregates have significant deficits in the acquisition phase of the object recognition test. Our results seem in some contrast to the evidence reported in $\mathrm{AD}$ patients, who are unable to store newly acquired information but preserve old memories, especially in early-stage of the disease. However, based on our results, it cannot be excluded that, in AD patients, the old events have occurred before the brain damage and this made possible the retention of retrieved material which has been successfully consolidated and stored into old memories for such events. On the other hand, in regard to the ability of sA $\beta$ to impair memory, possibly by inhibiting LTP, it has been hypothesized that the peptide may play a role in the process of physiological forgetfulness (Hasegawa, 2007). Interestingly, pathologies in which this physiological process seems to be impaired, such as posttraumatic stress disorder, are linked to increased risk of cognitive decline and AD-associated dementia (Weiner et al., 2014). The mechanism by which $s A \beta$ peptide induces consolidation/retrieval impairment is not clear yet, but the present results raise the possibility that the modulation of glutamatergic neurotransmission could be involved. Indeed, memory processing requires glutamatergic 
receptor activation (Kandel et al., 2014). It has been recently demonstrated that $\mathrm{sA} \beta$ dimers extracted from $\mathrm{AD}$ cortex induced their effects by perturbing glutamatergic synaptic transmission, being mGlu receptors required for the induction of LTD, while NMDA receptors were needed for spine loss (Shankar et al., 2008). Moreover, an association of $A \beta$ peptides with several receptors has been described in literature, and these receptors might act as substrates for the $A \beta$-mediated detrimental effects leading to the aforementioned functional cognitive alterations. Among others, glutamatergic receptors have been proposed as mediating the effect of $\mathrm{A} \beta$ on synaptic plasticity and memory (Klyubin et al., 2008; Ferreira and Klein, 2011). Consistent with this, sA $\beta$ enhances NMDA receptor agonist-induced delayed $\operatorname{cog}$ nitive dysfunction (Dornan et al., 1993; Nakamura et al., 2006). Soluble $A \beta$ has also been found to enhance glutamate release from microglia (Noda et al., 1999), isolated brain nerve terminals (Bobich et al., 2004) and brain slices (Arias et al., 1995; Chin et al., 2007; Puzzo et al., 2008; Kabogo et al., 2010). Accordingly, in our experimental conditions, we found a significant increase in glutamate release in PFC of treated rats. Such result is of particular interest in our research considering that it has been reported that PFC plays a crucial role in object recognition as well as in memory consolidation (Ragozzino et al., 2002; Xiang and Brown, 2004). Moreover, blocking synthesis of protein or NMDA receptors resulted in failure of consolidation of memory $24 \mathrm{~h}$ after familiarization phase (Akirav and Maroun, 2006). On the other hand, several reports suggest that NMDA receptors were closely associated with $\mathrm{A} \beta$ oligomer binding sites on neurons (De Felice et al., 2007; Lacor et al., 2007; Dewachter et al., 2009). Then, given the role of NMDA receptors in $\mathrm{A} \beta$-induced synaptic dysfunction, we directly tested the hypothesis that a NMDA receptor-blocking dose of memantine $(5 \mathrm{mg} / \mathrm{kg}$ i.p.), clinically used to treat $\mathrm{AD}$, could counteract the consolidation/retrieval failure observed in sA $\beta$-treated rats. Memantine is an NMDA receptor open channel blocker, which has been found to act with a low-to-moderate affinity at therapeutic concentrations (Lipton, 2007; Parsons and Gilling, 2007; Parsons et al., 2007). Unlike many high affinity antagonists (Ikonomidou and Turski, 2002), it preferentially blocks excessive or inappropriate activation of NMDA receptors, while leaving physiological NMDA receptor-mediated activity unaffected (Lipton, 2007; Parsons and Gilling, 2007; Parsons et al., 2007). In vitro and in vivo studies using animal models of neurodegenerative diseases and stroke showed that memantine protects neurons from NMDA receptor-mediated excitotoxic damage (De Felice et al., 2007; Xia et al., 2010). Mechanistically, we found that acute treatment with NMDA receptor blocking dose of memantine can prevent the consolidation/retrieval failure, caused by exogenous acute $\mathrm{sA} \beta$ administration. Accordingly, these results confirm previous data showing that sA $\beta$ oligomers directly activate NMDA receptors (Texidó et al., 2011). It should be noted that the observed effects of memantine in the present study were achieved at concentrations that are considered clinically relevant, leading to plasma levels of $1.2 \mu \mathrm{M}$, and that memantine has been shown to be active as an NMDA receptor antagonist at this concentrations as well (Misztal et al., 1996; Danysz and Parsons, 2003). Of note, when memantine was given before encoding memory process, animals remained cognitively impaired, suggesting that, in presence of exogenous $\mathrm{sA} \beta$, excessive NMDA activation, due to increased glutamate release, is deleterious only during the consolidation/retrieval phase. On the other hand, it has been demonstrated that, if acute administration is used, than memantine $5 \mathrm{mg} / \mathrm{kg}$ i.p., is the maximal therapeutically relevant dose for 30-60 min time point, although age, strain, gender and health status of rats should also been considered, since they can influence pharmacokinetic parameters considerably (Danysz et al., 1997). In addition, it has been reported that such dose took the peak serum concentration at ca. $30 \mathrm{~min}$, which correspond to the upper limit of dose seen in patients and volunteers after therapeutic dose of memantine (Zoladz et al., 2006). It is important to underline that, under our experimental conditions, memantine, given alone or co-administered with sA $\beta$ had no effect on locomotor (data not shown) and exploratory activities. Our findings are in good agreement with clinical results showing that memantine treatment improves cognitive performance in $\mathrm{AD}$ patients (Peskind et al., 2006). In addition, memantine administration has been previously associated to an improvement in cognitive performance in animal models of $\mathrm{AD}$, characterized by amyloid deposition (Nakamura et al., 2006; Martinez-Coria et al., 2010). The novelty of our findings relies on the evidence that memantine was effective in the absence of actual $\mathrm{A} \beta$ deposits. In this regard, we have previously demonstrated, by immunofluorescence experiments, that after 48 h from i.c.v. injection, sA $\beta$ peptide was no longer detectable in the ventricular space (Trabace et al., 2007). Then, it seems likely from our results that $\mathrm{sA} \beta$-induced impairment of consolidation/retrieval of long term memories and memantine ability to restore cognitive deficits might precede the development of insoluble amyloid plaque cores and then neurodegeneration. Our data also indicate that, in this model, acute sA $\beta$-mediated memory impairment is not dependent on a persistent neurodegenerative phenomenon and can be rescued, suggesting that early targeting $\mathrm{sA} \beta$-induced alterations might lead to the recovery of cognitive impairment.

\section{CONCLUSIONS}

Our study showed that sA $\beta$ was able to acutely alter consolidation/retrieval of long term memory, and this effect suggesting that early targeting $s A \beta$-induced alterations might lead to the recovery of cognitive was prevented by the use of a clinically relevant dose of memantine. This evidence suggests that $\mathrm{sA} \beta$ induced consolidation/retrieval failure might contribute to the memory impairment observed in $\mathrm{AD}$ even in the early stages of disease. Moreover, the present findings further our knowledge on the possibility of prevent cognitive deficits with NMDA receptor inhibition in an acute rat model of i.c.v. sA $\beta$ injection. Besides, using the acute animal model to explore the mechanism of action of sA $\beta$, it might also be helpful to assess the efficacy of novel therapeutic approaches directly aimed at this soluble species.

\section{ACKNOWLEDGMENTS}

This study was supported by PRIN 2011 (to Paolo Tucci) and PRIN 2012 (to Vincenzo Cuomo) from MIUR and by PON 0200186-2937475 to Luigia Trabace. 


\section{REFERENCES}

Akirav, I., and Maroun, M. (2006). Ventromedial prefrontal cortex is obligatory for consolidation and reconsolidation of object recognition memory. Cereb. Cortex 16, 1759-1765. doi: 10.1093/cercor/bhj114

Arendt, T. (2009). Synaptic degeneration in Alzheimer's disease. Acta Neuropathol. 118, 167-179. doi: 10.1007/s00401-009-0536-x

Arias, C., Arrieta, I., and Tapia, R. (1995). Beta-Amyloid peptide fragment 2535 potentiates the calcium-dependent release of excitatory amino acids from depolarized hippocampal slices. J. Neurosci. Res. 41, 561-566. doi: 10.1002/jnr. 490410416

Balducci, C., Beeg, M., Stravalaci, M., Bastone, A., Sclip, A., Biasini, E., et al. (2010). Synthetic amyloid-beta oligomers impair long-term memory independently of cellular prion protein. Proc. Natl. Acad. Sci. U S A 107, 2295-2300. doi: 10. 1073/pnas.0911829107

Berg, L., Mckeel, D. W. Jr., Miller, J. P., Storandt, M., Rubin, E. H., Morris, J. C., et al. (1998). Clinicopathologic studies in cognitively healthy aging and Alzheimer's disease: relation of histologic markers to dementia severity, age, sex and apolipoprotein E genotype. Arch. Neurol. 55, 326-335. doi: 10. 1001/archneur.55.3.326

Blennow, K., Bogdanovic, N., Alafuzoff, I., Ekman, R., and Davidsson, P. (1996). Synaptic pathology in Alzheimer's disease: relation to severity of dementia, but not to senile plaques, neurofibrillary tangles, or the ApoE4 allele. J. Neural Transm. 103, 603-618. doi: 10.1007/bf01273157

Bobich, J. A., Zheng, Q., and Campbell, A. (2004). Incubation of nerve endings with a physiological concentration of Abeta1-42 activates CaV2.2(N-Type)-voltage operated calcium channels and acutely increases glutamate and noradrenaline release. J. Alzheimers Dis. 6, 243-255.

Campagna, F., Catto, M., Purgatorio, R., Altomare, C. D., Carotti, A., De Stradis, A., et al. (2011). Synthesis and biophysical evaluation of arylhydrazono-1H-2indolinones as beta-amyloid aggregation inhibitors. Eur. J. Med. Chem. 46, 275284. doi: 10.1016/j.ejmech.2010.11.015

Canas, P. M., Simões, A. P., Rodrigues, R. J., and Cunha, R. A. (2014). Predominant loss of glutamatergic terminal markers in a beta-amyloid peptide model of Alzheimer's disease. Neuropharmacology 76(Pt. A), 51-56. doi: 10.1016/j. neuropharm.2013.08.026

Chin, J. H., Ma, L., Mactavish, D., and Jhamandas, J. H. (2007). Amyloid beta protein modulates glutamate-mediated neurotransmission in the rat basal forebrain: involvement of presynaptic neuronal nicotinic acetylcholine and metabotropic glutamate receptors. J. Neurosci. 27, 9262-9269. doi: 10. 1523/JNEUROSCI.1843-07.2007

Christensen, D. Z., Kraus, S. L., Flohr, A., Cotel, M. C., Wirths, O., and Bayer, T. A. (2008). Transient intraneuronal A beta rather than extracellular plaque pathology correlates with neuron loss in the frontal cortex of APP/PS1KI mice. Acta Neuropathol. 116, 647-655. doi: 10.1007/s00401-0080451-6

Clarke, J. R., Cammarota, M., Gruart, A., Izquierdo, I., and Delgado-Garcia, J. M. (2010). Plastic modifications induced by object recognition memory processing. Proc. Natl. Acad. Sci. U S A 107, 2652-2657. doi: 10.1073/pnas.0915 059107

Cleary, J. P., Walsh, D. M., Hofmeister, J. J., Shankar, G. M., Kuskowski, M. A., Selkoe, D. J., et al. (2005). Natural oligomers of the amyloid-beta protein specifically disrupt cognitive function. Nat. Neurosci. 8, 79-84. doi: 10.1038/ nn 1372

Colaianna, M., Tucci, P., Zotti, M., Morgese, M. G., Schiavone, S., Govoni, S., et al. (2010). Soluble beta amyloid(1-42): a critical player in producing behavioural and biochemical changes evoking depressive-related state? Br. J. Pharmacol. 159, 1704-1715. doi: 10.1111/j.1476-5381.2010. 00669.x

Cowburn, R. F., Wiehager, B., Trief, E., Li-Li, M., and Sundström, E. (1997). Effects of beta-amyloid-(25-35) peptides on radioligand binding to excitatory amino acid receptors and voltage-dependent calcium channels: evidence for a selective affinity for the glutamate and glycine recognition sites of the NMDA receptor. Neurochem. Res. 22, 1437-1442. doi: 10.1023/A:10219421 09490

Danysz, W., and Parsons, C. G. (2003). The NMDA receptor antagonist memantine as a symptomatological and neuroprotective treatment for Alzheimer's disease: preclinical evidence. Int. J. Geriatr. Psychiatry 18, S23-S32. doi: 10.1002/ gps.938
Danysz, W., Parsons, C. G., Kornhuber, J., Schmidt, W. J., and Quack, G. (1997). Aminoadamantanes as NMDA receptor antagonists and antiparkinsonian agents-preclinical studies. Neurosci. Biobehav. Rev. 21, 455-468. doi: 10. 1016/s0149-7634(96)00037-1

De Felice, F. G., Velasco, P. T., Lambert, M. P., Viola, K., Fernandez, S. J., Ferreira, S. T., et al. (2007). Abeta oligomers induce neuronal oxidative stress through an N-methyl-D-aspartate receptor-dependent mechanism that is blocked by the Alzheimer drug memantine. J. Biol. Chem. 282, 11590-11601. doi: 10.1074/jbc. m607483200

Dewachter, I., Filipkowski, R. K., Priller, C., Ris, L., Neyton, J., Croes, S., et al. (2009). Deregulation of NMDA-receptor function and down-stream signaling in APP[V717I] transgenic mice. Neurobiol. Aging 30, 241-256. doi: 10.1016/j. neurobiolaging.2007.06.011

Dornan, W. A., Kang, D. E., Mccampbell, A., and Kang, E. E. (1993). Bilateral injections of beta $\mathrm{A}(25-35)+\mathrm{IBO}$ into the hippocampus disrupts acquisition of spatial learning in the rat. Neuroreport 5, 165-168. doi: 10.1097/00001756199311180-00018

Ennaceur, A., and Delacour, J. (1988). A new one-trial test for neurobiological studies of memory in rats. 1: behavioral data. Behav. Brain Res. 31, 47-59. doi: 10.1016/0166-4328(88)90157-x

Ferreira, S. T., and Klein, W. L. (2011). The Abeta oligomer hypothesis for synapse failure and memory loss in Alzheimer's disease. Neurobiol. Learn. Mem. 96, 529543. doi: 10.1016/j.nlm.2011.08.003

Giannakopoulos, P., Herrmann, F. R., Bussiere, T., Bouras, C., Kovari, E., Perl, D. P., et al. (2003). Tangle and neuron numbers, but not amyloid load, predict cognitive status in Alzheimer's disease. Neurology 60, 1495-1500. doi: 10.1212/01.wnl. 0000063311.58879 .01

Giannakopoulos, P., Kövari, E., Gold, G., Von Gunten, A., Hof, P. R., and Bouras, C. (2009). Pathological substrates of cognitive decline in Alzheimer's disease. Front. Neurol. Neurosci. 24, 20-29. doi: 10.1159/000197881

Giustino, A., Beckett, S., Ballard, T., Cuomo, V., and Marsden, C. A. (1996). Perinatal cocaine reduces responsiveness to cocaine and causes alterations in exploratory behavior and visual discrimination in young-adult rats. Brain Res. 728, 149-156. doi: 10.1016/s0006-8993(96)00229-6

Haass, C., and Selkoe, D. J. (2007). Soluble protein oligomers in neurodegeneration: lessons from the Alzheimer's amyloid beta-peptide. Nat. Rev. Mol. Cell Biol. 8, 101-112. doi: 10.1038/nrm2101

Hasegawa, T. (2007). Prolonged stress will induce Alzheimer's disease in elderly people by increased release of homocysteic acid. Med. Hypotheses 69, 1135-1139. doi: 10.1016/j.mehy.2007.02.034

Ikonomidou, C., and Turski, L. (2002). Why did NMDA receptor antagonists fail clinical trials for stroke and traumatic brain injury? Lancet Neurol. 1, 383-386. doi: 10.1016/s1474-4422(02)00164-3

Jin, M., Shepardson, N., Yang, T., Chen, G., Walsh, D., and Selkoe, D. J. (2011). Soluble amyloid beta-protein dimers isolated from Alzheimer cortex directly induce Tau hyperphosphorylation and neuritic degeneration. Proc. Natl. Acad. Sci. U S A 108, 5819-5824. doi: 10.1073/pnas.101703 3108

Kabogo, D., Rauw, G., Amritraj, A., Baker, G., and Kar, S. (2010). SS-amyloidrelated peptides potentiate $\mathrm{K}+$-evoked glutamate release from adult rat hippocampal slices. Neurobiol. Aging 31, 1164-1172. doi: 10.1016/j.neurobiolaging. 2008.08.009

Kandel, E. R., Dudai, Y., and Mayford, M. R. (2014). The molecular and systems biology of memory. Cell 157, 163-186. doi: 10.1016/j.cell.2014. 03.001

Kelly, B. L., and Ferreira, A. (2006). Beta-Amyloid-induced dynamin 1 degradation is mediated by $\mathrm{N}$-methyl-D-aspartate receptors in hippocampal neurons. J. Biol. Chem. 281, 28079-28089. doi: 10.1074/jbc.m605081200

Klyubin, I., Betts, V., Welzel, A. T., Blennow, K., Zetterberg, H., Wallin, A., et al. (2008). Amyloid beta protein dimer-containing human CSF disrupts synaptic plasticity: prevention by systemic passive immunization. J. Neurosci. 28, 42314237. doi: 10.1523/JNEUROSCI.5161-07.2008

Kowalewski, T., and Holtzman, D. M. (1999). In situ atomic force microscopy study of Alzheimer's beta-amyloid peptide on different substrates: new insights into mechanism of beta-sheet formation. Proc. Natl. Acad. Sci. U S A 96, 3688-3693. doi: 10.1073/pnas.96.7.3688

Lacor, P. N., Buniel, M. C., Furlow, P. W., Clemente, A. S., Velasco, P. T., Wood, M., et al. (2007). Abeta oligomer-induced aberrations in synapse composition, 
shape and density provide a molecular basis for loss of connectivity in Alzheimer's disease. J. Neurosci. 27, 796-807. doi: 10.1523/jneurosci.3501-06. 2007

Lambert, M. P., Barlow, A. K., Chromy, B. A., Edwards, C., Freed, R., Liosatos, M., et al. (1998). Diffusible, nonfibrillar ligands derived from Abeta1-42 are potent central nervous system neurotoxins. Proc. Natl. Acad. Sci. U S A 95, 6448-6453. doi: 10.1073/pnas.95.11.6448

Lesné, S., Koh, M. T., Kotilinek, L., Kayed, R., Glabe, C. G., Yang, A., et al. (2006). A specific amyloid-beta protein assembly in the brain impairs memory. Nature 440, 352-357. doi: 10.1038/nature04533

Lipton, S. A. (2007). Pathologically-activated therapeutics for neuroprotection: mechanism of NMDA receptor block by memantine and Snitrosylation. Curr. Drug Targets 8, 621-632. doi: 10.2174/13894500778061 8472

Lopez-Ramos, J. C., Jurado-Parras, M. T., Sanfeliu, C., Acuna-Castroviejo, D., and Delgado-Garcia, J. M. (2012). Learning capabilities and CA1prefrontal synaptic plasticity in a mice model of accelerated senescence. Neurobiol. Aging 33, 627.e13-627.e26. doi: 10.1016/j.neurobiolaging.2011. 04.005

Lue, L. F., Kuo, Y. M., Roher, A. E., Brachova, L., Shen, Y., Sue, L., et al. (1999). Soluble amyloid beta peptide concentration as a predictor of synaptic change in Alzheimer's disease. Am. J. Pathol. 155, 853-862. doi: 10.1016/s00029440(10)65184-X

Mangialasche, F., Solomon, A., Winblad, B., Mecocci, P., and Kivipelto, M. (2010). Alzheimer's disease: clinical trials and drug development. Lancet Neurol. 9, 702716. doi: 10.1016/S1474-4422(10)70119-8

Markowitsch, H. J., and Staniloiu, A. (2012). Amnesic disorders. Lancet 380, 14291440. doi: 10.1016/S0140-6736(11)61304-4

Martinez-Coria, H., Green, K. N., Billings, L. M., Kitazawa, M., Albrecht, M., Rammes, G., et al. (2010). Memantine improves cognition and reduces Alzheimer's-like neuropathology in transgenic mice. Am. J. Pathol. 176, 870880. doi: 10.2353/ajpath.2010.090452

Mayford, M., Siegelbaum, S. A., and Kandel, E. R. (2012). Synapses and memory storage. Cold Spring Harb. Perspect. Biol. 4:a005751. doi: 10.1101/cshperspect. a005751

McLean, C. A., Cherny, R. A., Fraser, F. W., Fuller, S. J., Smith, M. J., Beyreuther, K., et al. (1999). Soluble pool of Abeta amyloid as a determinant of severity of neurodegeneration in Alzheimer's disease. Ann. Neurol. 46, 860-866. doi: 10.1002/1531-8249(199912)46:6<860::aid-ana8>3.0. $\mathrm{co} ; 2-\mathrm{m}$

Misztal, M., Frankiewicz, T., Parsons, C. G., and Danysz, W. (1996). Learning deficits induced by chronic intraventricular infusion of quinolinic acidprotection by MK-801 and memantine. Eur. J. Pharmacol. 296, 1-8. doi: 10. 1016/0014-2999(95)00682-6

Molnár, Z., Soós, K., Lengyel, I., Penke, B., Szegedi, V., and Budai, D. (2004). Enhancement of NMDA responses by beta-amyloid peptides in the hippocampus in vivo. Neuroreport 15, 1649-1652. doi: 10.1097/01.wnr.0000134471. 06244.d2

Morgese, M. G., Tucci, P., Colaianna, M., Zotti, M., Cuomo, V., Schiavone, S., et al. (2014). Modulatory activity of soluble beta amyloid on HPA axis function in rats. Curr. Pharm. Des. 20, 2539-2546. doi: 10.2174/138161281131999 90500

Mucke, L., and Selkoe, D. J. (2012). Neurotoxicity of amyloid beta-protein: synaptic and network dysfunction. Cold Spring Harb. Perspect. Med. 2:a006338. doi: 10. 1101/cshperspect.a006338

Nakamura, S., Murayama, N., Noshita, T., Katsuragi, R., and Ohno, T. (2006). Cognitive dysfunction induced by sequential injection of amyloidbeta and ibotenate into the bilateral hippocampus; protection by memantine and MK-801. Eur. J. Pharmacol. 548, 115-122. doi: 10.1016/j.ejphar.2006. 07.049

Noda, M., Nakanishi, H., and Akaike, N. (1999). Glutamate release from microglia via glutamate transporter is enhanced by amyloid-beta peptide. Neuroscience 92, 1465-1474. doi: 10.1016/s0306-4522(99)00036-6

Parsons, C. G., and Gilling, K. (2007). Memantine as an example of a fast, voltagedependent, open channel N-methyl-D-aspartate receptor blocker. Methods Mol. Biol. 403, 15-36. doi: 10.1007/978-1-59745-529-9_2

Parsons, C. G., Stoffler, A., and Danysz, W. (2007). Memantine: a NMDA receptor antagonist that improves memory by restoration of homeostasis in the glutamatergic system-too little activation is bad, too much is even worse. Neuropharmacology 53, 699-723. doi: 10.1016/j.neuropharm.2007. 07.013

Paxinos, G., and Watson, C. (1998). The Rat Brain in Stereotaxic Coordinates. New York: Academic Press.

Peskind, E. R., Potkin, S. G., Pomara, N., Ott, B. R., Graham, S. M., Olin, J. T., et al. (2006). Memantine treatment in mild to moderate Alzheimer disease: a 24-week randomized, controlled trial. Am. J. Geriatr. Psychiatry 14, 704-715. doi: 10.1097/01.JGP.0000224350.82719.83

Poling, A., Morgan-Paisley, K., Panos, J. J., Kim, E. M., O’hare, E., Cleary, J. P., et al. (2008). Oligomers of the amyloid-beta protein disrupt working memory: confirmation with two behavioral procedures. Behav. Brain Res. 193, 230-234. doi: 10.1016/j.bbr.2008.06.001

Puoliväli, J., Wang, J., Heikkinen, T., Heikkilä, M., Tapiola, T., Van Groen, T., et al. (2002). Hippocampal A beta 42 levels correlate with spatial memory deficit in APP and PS1 double transgenic mice. Neurobiol. Dis. 9, 339-347. doi: 10. 1006/nbdi.2002.0481

Puzzo, D., Privitera, L., Leznik, E., Fà, M., Staniszewski, A., Palmeri, A., et al. (2008). Picomolar amyloid-beta positively modulates synaptic plasticity and memory in hippocampus. J. Neurosci. 28, 14537-14545. doi: 10.1523/jneurosci.2692-08. 2008

Ragozzino, M. E., Detrick, S., and Kesner, R. P. (2002). The effects of prelimbic and infralimbic lesions on working memory for visual objects in rats. Neurobiol. Learn. Mem. 77, 29-43. doi: 10.1006/nlme.2001.4003

Shankar, G. M., Li, S., Mehta, T. H., Garcia-Munoz, A., Shepardson, N. E., Smith, I., et al. (2008). Amyloid-beta protein dimers isolated directly from Alzheimer's brains impair synaptic plasticity and memory. Nat. Med. 14, 837-842. doi: 10. $1038 / \mathrm{nm} 1782$

Sokolow, S., Henkins, K. M., Bilousova, T., Miller, C. A., Vinters, H. V., Poon, W., et al. (2012). AD synapses contain abundant Abeta monomer and multiple soluble oligomers, including a 56-kDa assembly. Neurobiol. Aging 33, 15451555. doi: 10.1016/j.neurobiolaging.2011.05.011

Taglialatela, G., Hogan, D., Zhang, W. R., and Dineley, K. T. (2009). Intermediateand long-term recognition memory deficits in $\mathrm{Tg} 2576$ mice are reversed with acute calcineurin inhibition. Behav. Brain Res. 200, 95-99. doi: 10.1016/j.bbr. 2008.12.034

Texidó, L., Martin-Satué, M., Alberdi, E., Solsona, C., and Matute, C. (2011). Amyloid beta peptide oligomers directly activate NMDA receptors. Cell Calcium 49, 184-190. doi: 10.1016/j.ceca.2011.02.001

Trabace, L., Kendrick, K. M., Castrignanò, S., Colaianna, M., De Giorgi, A., Schiavone, S., et al. (2007). Soluble amyloid beta1-42 reduces dopamine levels in rat prefrontal cortex: relationship to nitric oxide. Neuroscience 147, 652-663. doi: 10.1016/j.neuroscience.2007.04.056

Watanabe, T., Yamagata, N., Takasaki, K., Sano, K., Hayakawa, K., Katsurabayashi, S., et al. (2009). Decreased acetylcholine release is correlated to memory impairment in the Tg2576 transgenic mouse model of Alzheimer's disease. Brain Res. 1249, 222-228. doi: 10.1016/j.brainres.2008. 10.029

Weiner, M. W., Veitch, D. P., Hayes, J., Neylan, T., Grafman, J., Aisen, P. S., et al. (2014). Effects of traumatic brain injury and posttraumatic stress disorder on Alzheimer's disease in veterans, using the Alzheimer's disease neuroimaging initiative. Alzheimers Dement. 10, S226-S235. doi: 10.1016/j.jalz.2014. 04.005

Wilcox, K. C., Lacor, P. N., Pitt, J., and Klein, W. L. (2011). Abeta oligomer-induced synapse degeneration in Alzheimer's disease. Cell. Mol. Neurobiol. 31, 939-948. doi: 10.1007/s10571-011-9691-4

Wiltfang, J., Smirnov, A., Schnierstein, B., Kelemen, G., Matthies, U., Klafki, H. W., et al. (1997). Improved electrophoretic separation and immunoblotting of betaamyloid (A beta) peptides 1-40, 1-42 and 1-43. Electrophoresis 18, 527-532. doi: 10.1002/elps.1150180332

Xia, P., Chen, H. S., Zhang, D., and Lipton, S. A. (2010). Memantine preferentially blocks extrasynaptic over synaptic NMDA receptor currents in hippocampal autapses. J. Neurosci. 30, 11246-11250. doi: 10.1523/jneurosci.2488-10. 2010

Xiang, J. Z., and Brown, M. W. (2004). Neuronal responses related to long-term recognition memory processes in prefrontal cortex. Neuron 42, 817-829. doi: 10 . 1016/j.neuron.2004.05.013

Yu, L., Edalji, R., Harlan, J. E., Holzman, T. F., Lopez, A. P., Labkovsky, B., et al. (2009). Structural characterization of a soluble amyloid beta-peptide oligomer. Biochemistry 48, 1870-1877. doi: 10.1021/bi802046n 
Zhang, W., Bai, M., Xi, Y., Hao, J., Liu, L., Mao, N., et al. (2012). Early memory deficits precede plaque deposition in APPswe/PS1dE9 mice: involvement of oxidative stress and cholinergic dysfunction. Free Radic. Biol. Med. 52, 1443-1452. doi: 10.1016/j.freeradbiomed.2012. 01.023

Zoladz, P. R., Campbell, A. M., Park, C. R., Schaefer, D., Danysz, W., and Diamond, D. M. (2006). Enhancement of long-term spatial memory in adult rats by the noncompetitive NMDA receptor antagonists, memantine and neramexane. Pharmacol. Biochem. Behav. 85, 298-306. doi: 10.1016/j.pbb.2006. 08.011

Conflict of Interest Statement: The authors declare that the research was conducted in the absence of any commercial or financial relationships that could be construed as a potential conflict of interest.
Received: 15 July 2014; accepted: 04 September 2014; published online: 19 September 2014.

Citation: Tucci P, Mhillaj E, Morgese MG, Colaianna M, Zotti M, Schiavone S, Cicerale M, Trezza V, Campolongo P, Cuomo V and Trabace L (2014) Memantine prevents memory consolidation failure induced by soluble beta amyloid in rats. Front. Behav. Neurosci. 8:332. doi: 10.3389/fnbeh.2014.00332

This article was submitted to the journal Frontiers in Behavioral Neuroscience. Copyright (C) 2014 Tucci, Mhillaj, Morgese, Colaianna, Zotti, Schiavone, Cicerale, Trezza, Campolongo, Cuomo and Trabace. This is an open-access article distributed under the terms of the Creative Commons Attribution License (CC BY). The use, distribution or reproduction in other forums is permitted, provided the original author(s) or licensor are credited and that the original publication in this journal is cited, in accordance with accepted academic practice. No use, distribution or reproduction is permitted which does not comply with these terms. 